\title{
Myriad mysteries of glucose homeostasis
}

\section{Hideki Katagiri ${ }^{1}$}

Received: 13 December 2017 / Published online: 24 January 2018

(C) The Japan Diabetes Society 2018
In our bodies, glucose metabolism is maintained in an amazingly elaborate manner, in both postprandial and fasting states, than ever previously imagined. The widely used continuous glucose monitoring system has revealed that a not inconsiderable population with normal glucose tolerance shows slight fluctuations in glucose levels even after eating. We believe that insulin is secreted by pancreatic $\beta$ cells in response to blood glucose elevation, resulting in lowering of blood glucose to normal levels. That is to say, based on this mechanism, insulin secretion is stimulated AFTER blood glucose elevation. In a portion of normal subjects, however, blood glucose elevation would not initially occur even after food intake. Thus, glucose-stimulated insulin secretion cannot, by itself, explain these observations. Therefore, I have come to believe that there must be predictive and preventive mechanisms which function prior to blood glucose elevation.

Considering that the blood volume of an adult human is approximately 51 , a blood glucose concentration of $100 \mathrm{mg} /$ $\mathrm{dl}$ means that the total glucose amounts in blood is only $5 \mathrm{~g}$ at the whole-blood level. Without blood glucose lowering systems, $100 \mathrm{~g}$ of carbohydrate from dietary intake is estimated to raise blood glucose levels by $2000 \mathrm{mg} / \mathrm{dl}$. This indicates that blood has minimal functional capacity as a glucose buffer. What, then, is the main mechanism maintaining blood glucose levels with little fluctuation after we eat?

Dietary carbohydrates do not rapidly and directly flow into the peripheral circulation. First, they are digested and absorbed in the gut and enter the portal vein. This step may assure gradual glucose entry rates. Next, the portal blood flow goes through the liver, where a substantial amount of glucose is metabolized. Only the residuals reach the systemic circulation. In addition, many red blood cells within the blood may take up glucose, possibly playing a buffering function. If blood glucose levels rise even after all of these

\section{Hideki Katagiri}

katagiri@med.tohoku.ac.jp

1 Department of Metabolism and Diabetes, Tohoku University Graduate School of Medicine, 2-1 Seiryo, Aoba-ku, Sendai, Miyagi 980-8575, Japan steps, the increased glucose within the pancreatic circulation, accompanied by incretin signals, stimulates insulin secretion from pancreatic $\beta$ cells, a process we refer to as glucose-stimulated insulin secretion. Therefore, it is possible that these aforementioned mechanisms which function prior to glucose-stimulated insulin secretion play important roles in maintaining glucose homeostasis. However, as we have found in our clinical experience, it is common for patients with type 1 diabetes to show massive elevations in blood glucose when they do not inject insulin prior to betweenmeal snacking. Therefore, it has been speculated that these mechanisms functioning prior to insulin secretion alone are not sufficient to minimize blood glucose fluctuations. Thus, we must assume the existence of a pre-conditioning mechanism which predicts the impending risk of blood glucose elevation and prepares the physiological conditions for preventing hyperglycemia. I wonder whether undiscovered systems, including neuronal networks, must function as such a predictive and preventive mechanism(s).

Investigation of diabetes and/or managing patients with diabetes has focused on considerable attention on study of the mechanisms lowering blood glucose levels. On the other hand, endogenous mechanisms preventing hypoglycemia during fasting are also filled with physiological and clinical mysteries. In fasting states, glycogenolysis, followed by enhanced gluconeogenesis, mainly by hepatocytes, is well known to inhibit blood glucose lowering.

Blood glucose levels during the fasting periods, particularly while sleeping, are well known to be very stable in people with normal glucose tolerance. All cells in the organs/tissues throughout the body take up and metabolize glucose as an energy source to sustain life. Even under the fasting conditions, of course, this is true. Moreover, since the buffering function of the blood is quite small, as mentioned above, excesses and deficiencies of hepatic glucose release would directly induce hyper- and hypo-glycemia. These observations mean that hepatocytes release exactly the same amounts of glucose in total as those which are utilized throughout the body. Thus, the liver somehow knows how much glucose is consumed by remote organs in 
a real-time manner and continually produces and releases the right amounts of glucose. This mechanism requires great accuracy in terms of both timing and the amounts of glucose released. It is also surprising that treatment with sodium glucose cotransporter 2 inhibitors (SGLT2i) alone rarely causes hypoglycemia even in fasting states. SGLT2i removes glucose from the blood as urinary glucose at a rate of $3-4 \mathrm{~g} / \mathrm{h}$. These glucose amounts are considerable, given that the entire volume of blood in the body contains about $5 \mathrm{~g}$ of glucose. Thus, the liver must continually sense the extra amounts of urinary glucose and rapidly produce glucose at markedly higher rates in response to SGLT2i treatment.

By what mechanism does this elaborate regulation involving both the timing and amounts of glucose release become reality? It is well known that gluconeogenesis in hepatocytes is mainly regulated by insulin and glucagon. The proposed main mechanism involves regulating the expressions of key enzymes, such as phosphoenolpyruvate carboxykinase (PEPCK) and glucose-6-phosphatase (G6Pase), at their mRNA levels. However, I speculate that these alterations at the mRNA expression levels cannot achieve the instantaneous regulation. These extremely elaborate regulatory processes, with neither discernible time lag nor over/undershooting of the required glucose amounts, may also involve as yet unknown inter-organ mechanisms, such as neuronal networks, which "supervise" whole body metabolism.

Taken together, mechanisms maintaining systemic glucose homeostasis seem to be far from being fully understood. Furthermore, these exquisite mechanisms that underlie normal glucose conditions may be good targets for elucidating the causes of, and thereby novel treatments for, diabetes. What a mysterious and fascinating world they provide for conducting research on systemic regulation of glucose metabolism! 